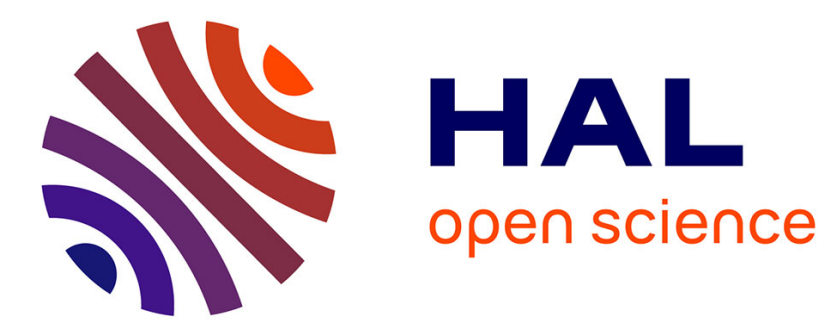

\title{
A variational model for thin structure segmentation based on a directional regularization
}

Odyssée Merveille, Olivia Miraucourt, Stéphanie Salmon, Nicolas Passat, Hugues Talbot

\section{- To cite this version:}

Odyssée Merveille, Olivia Miraucourt, Stéphanie Salmon, Nicolas Passat, Hugues Talbot. A variational model for thin structure segmentation based on a directional regularization. International Conference on Image Processing (ICIP), 2016, Phoenix, United States. pp.4324-4328, 10.1109/ICIP.2016.7533176 . hal-01472645

\section{HAL Id: hal-01472645 \\ https://hal.science/hal-01472645}

Submitted on 21 Feb 2017

HAL is a multi-disciplinary open access archive for the deposit and dissemination of scientific research documents, whether they are published or not. The documents may come from teaching and research institutions in France or abroad, or from public or private research centers.
L'archive ouverte pluridisciplinaire HAL, est destinée au dépôt et à la diffusion de documents scientifiques de niveau recherche, publiés ou non, émanant des établissements d'enseignement et de recherche français ou étrangers, des laboratoires publics ou privés. 


\title{
A VARIATIONAL MODEL FOR THIN STRUCTURE SEGMENTATION BASED ON A DIRECTIONAL REGULARIZATION
}

\author{
Odyssée Merveille ${ }^{1,3}$, Olivia Miraucourt ${ }^{1,2}$, Stéphanie Salmon ${ }^{2}$, Nicolas Passat $^{3}$, Hugues Talbot $^{1}$ \\ ${ }^{1}$ Université Paris-Est - ESIEE, LIGM, UMR CNRS 8049, France \\ ${ }^{2,3}$ Université de Reims Champagne-Ardenne, ${ }^{2} \mathrm{LMR},{ }^{3} \mathrm{CReSTIC}$, France
}

\begin{abstract}
Tubular structure segmentation is an important task, with many applications in medical image analysis such as vessel segmentation both in 2D and 3D. However, this task is challenging due to the spatial sparsity of these objects, implying a high sensitivity to noise.

An important cue in this context is the local orientation of the tubular structures. Using this information, it is possible to regularize the structures without destroying its integrity. In this article, we take advantage of recent advances in orientation estimation to propose a directional regularization prior for tubular structures, suitable for use in a variational framework. We illustrate on both synthetic and 2D real data.
\end{abstract}

Index Terms - Total variation, weighted total variation, ChanVese model, segmentation.

\section{INTRODUCTION}

Thin structures in $n \mathrm{D}$ images are characterized by a significantly smaller size in at least one of their $n$ dimensions. 1D thin structures correspond to line-like or tube-like objects respectively in $2 \mathrm{D}$ or 3D images. In the following, we always refer to tubular structures independently of the image dimension. Extraction of tubular structures (vessels, neurones, fibres, ...) is a mandatory but challenging task for many biomedical applications. In this context, angiographic imaging - i.e., the imaging of vessels - received a particular attention [1]; the reader is also referred to [2] for a more general survey on tubular structure analysis.

Tubular structures are among the hardest to handle in image processing. The difficulties derive from their spatial sparsity, their small size (often only a few pixels thick) and their complex geometric properties (thin, long, curvilinear, ...). Indeed, all these properties imply that such structures are naturally fragile and their detection is often disturbed by noise and other artifacts.

In this work, we propose to combine a variational approach, that has been proved successful in image restoration problems, with novel priors specific to tubular structures. Mathematically speaking, image restoration is an ill-posed inverse problem; to be able to solve it, we must impose some regularity on the solution. Tikhonov [3] proposed a quadratic regularization term; however, this generates strong blurring effects. In 1992, Rudin, Osher and Fatemi (ROF) replaced this quadratic regularization with an $\ell^{1}$ norm of the gradient, called total variation, which better preserves edges [4]. By constraining the output image to take a limited set of values, this

This research was partially funded by a grant from the Région Champagne-Ardenne and by the French Agence Nationale de la Recherche (Grant Agreement ANR-12-MONU-0010). restoration framework can be used for segmentation. In particular, the Chan-Vese model [5] divides the image into two regions of piecewise-constant intensities. If these two piecewise constant values are known, it results in a convex problem that can be solved exactly [6].

Amongst all the tubular structure filters in the literature, Frangi vesselness [7] is currently considered as the gold standard. From this second derivative-based filter, we can extract two features: (i) a vesselness feature which gives, for each image pixel, the probability to belong to a tubular structure, and (ii) a direction feature that gives, for each image pixel, the direction (as a vector) of the associated tubular structure. Recently, we introduced the RORPO operator $[8,9]$ which is a robust alternative to the Frangi filter, which also provides both vesselness and direction features.

In the context of tubular structures, extensions of the Chan-Vese model have been proposed by adding tubular priors, e.g., superellipsoids [10], B-splines framelet [11], adaptive dictionaries [12] and elastical regularization [13]. In [14], we also proposed a variational approach for tubular structure restoration. Indeed, by considering the Frangi vesselness feature, we designed an adaptive regularization parameter to avoid the intensity loss in tubular structures, which is a characteristic of classical variational restoration frameworks.

In this article, we propose a new regularization term for variational segmentation. This term embeds both vesselness and direction information. The main advantage of this new regularization term is that, by regularizing along the tubular structure axis, we do not only avoid the intensity loss in tubular structures, but we also induce reconnections while preserving the required isotropic regularization in the background.

\section{SEGMENTATION MODEL}

\subsection{General problem and notations}

Let $X=\mathbb{R}^{N \times N}$ be the Euclidean vector space and $f$ be the $2 \mathrm{D}$ original image in $X$ of size $N \times N$. We consider the general minimization problem:

$$
\min _{u \in X} F(u, f)+\lambda G(u)
$$

where $F$ the data fidelity term and $G$ the regularization term. $\lambda \in \mathbb{R}$ is a weighting parameter.

A common regularization term $G$ in such variational segmentation problem is the total variation (TV) which is defined by:

$$
\operatorname{TV}(u)=\|\nabla u\|_{2,1}=\sum_{0 \leq i, j<N} \sqrt{\left((\nabla u)_{i, j}^{x}\right)^{2}+\left((\nabla u)_{i, j}^{y}\right)^{2}}
$$

where $\nabla u=\left((\nabla u)^{x},(\nabla u)^{y}\right)$ is the $2 \mathrm{D}$ gradient. 


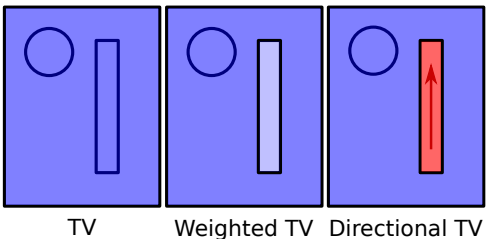

(a)

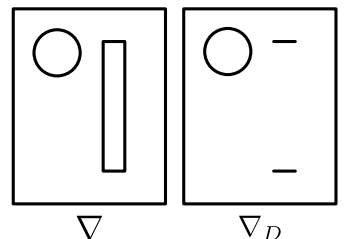

(b)
Fig. 1: (a) Regularization principle in the case of the classical TV (left), the weighted-TV [14] (middle) and our directional TV (right). The brighter the color, the higher the regularization. Non-tubular structures are not affected by the directional regularization. (b) The regular gradient $\nabla$ of the TV (left) and the directional gradient $\nabla_{D}$ (right) of our directional TV.

\subsection{Directional regularization principle}

In terms of tubular structure segmentation, the TV regularization (i.e. minimizing the norm of the gradient) is not well adapted since it penalizes contours. Thin tube-like structures are essentially composed of edges and so, are highly penalized. The TV regularization tends to remove them.

To cope with this problem, Miraucourt et al. [14] proposed a weighted TV scheme that includes the Frangi vesselness as a tubular prior. They used the vesselness to know where a tubular structure is likely to be. They proposed to regularize more strongly outside the tubular structure than inside. This strategy generally effectively prevents tubular structures from disappearing, however noise is not dealt with inside these structures, leading to many disconnections, and the smaller tubular structures are lost.

In this article, we propose a more suitable regularization term for tubular structure segmentation, by considering both the vesselness and direction features. Instead of regularizing less inside tubular structures, we keep a strong regularization but solely along the local tubular structures axis. In other words, we propose an intensity and directional spatially variant regularization term (see Fig. 1a).

\subsection{Directional total variation}

We now define a directional gradient operator $\nabla_{D}$ embedding the vesselness and directional information.

Peyré et al. [15] proposed a gradient computed on a weighted graph, generalized in [16]. Both the graph and its weights are computed, based on the initial image at each step of the optimization algorithm, resulting in an algorithm which may be time-consuming. In the present article, we propose a directional TV in which both graph and weights are computed from the direction and vesselness feature only once, at the initial stage of the process. This results in a regularization that is still adapted to tubular structures but more efficient in terms of complexity.

To define this directional gradient, $\nabla_{D} \in X^{p}$, we first define a span $\left(\mathrm{v}_{1}, \ldots, \mathrm{v}_{p}\right)$ of $p$ unitary vectors. This span contains all the discrete undirected orientations in a $k \times k$ neighborhood (see Fig. 2), thus $p=2\left(n^{2}-n+1\right)$ with $n=\frac{k-1}{2}$.

We can now define the directional gradient, in the direction of vectors $\left(\mathrm{v}_{i}\right)_{i \in \llbracket 1, p \rrbracket}$ :

$$
\begin{aligned}
\nabla_{D} u & =\left(D^{1} \circ\left(\nabla_{d} u\right)^{1}, \cdots, D^{p} \circ\left(\nabla_{d} u\right)^{1}\right) \\
\left(\nabla_{D} u\right)_{i, j} & =D_{i, j}^{1}\left(\nabla_{d} u\right)_{i, j}^{1} \mathrm{v}_{1}+\cdots+D_{i, j}^{p}\left(\nabla_{d} u\right)_{i, j}^{p} \mathrm{v}_{p}
\end{aligned}
$$

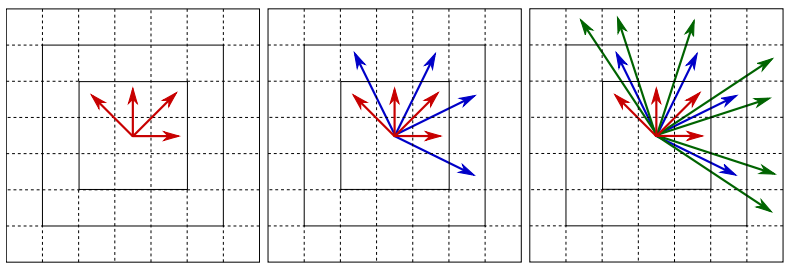

(a) $n=1$

(b) $n=2$

(c) $n=3$

with $D^{q} \in X, 1 \leq q \leq p$ a weight image such that:

$$
\begin{aligned}
& D_{i, j}^{q}=d_{i, j}^{q} \Phi_{i, j}+\left(1-\Phi_{i, j}\right) \\
& \sum_{q=1}^{p} D_{i, j}^{q}=1 \quad \forall(i, j) \in \llbracket 0, N \llbracket^{2}
\end{aligned}
$$

$\Phi \in X$ is a vesselness feature normalized to the interval $[0,1]$ and the directions coefficients, $\left(d^{i}\right)_{i \in \llbracket 1, p \rrbracket}$, are computed from the direction feature (see Sec. 3.1).

Intuitively, if $\Phi_{i, j}=0$, meaning the pixel $(i, j)$ does not belong to a tubular structure, then $\left(\nabla_{D} u\right)_{i, j}=\left(\left(\nabla_{d} u^{1}\right)_{i, j}, \ldots,\left(\nabla_{d} u^{p}\right)_{i, j}\right)$ is an isotropic gradient, resulting in an isotropic regularization. Conversely, if $\Phi_{i, j}=1$, meaning the pixel $(i, j)$ does belong to a tubular structure, then, $\left(\nabla_{D} u\right)_{i, j}=\left(d_{i, j}^{1}\left(\nabla_{d} u^{1}\right)_{i, j}, \ldots, d_{i, j}^{p}\left(\nabla_{d} u^{p}\right)_{i, j}\right)$ is a directional gradient, resulting in a directional regularization along the tubular structure axis.

Optimizing this new gradient is more satisfactory as only the extremal contours of the tubular structure appear in the directional gradient (see Fig.1.b). Consequently, optimizing our directional TV no longer induces the loss of tubular structure edges, while still regularizing inside the tubular structure.

\subsection{Model}

We propose a segmentation model based on the directional total variation, $\mathrm{TV}_{d}$, defined as follows:

$$
\underset{u \in[0,1]^{N \times N}}{\operatorname{minimize}}\left\langle c_{f}, u\right\rangle_{F}+\lambda\left\|\nabla_{D} u\right\|_{2,1}
$$

- $\left\|\nabla_{D} u\right\|_{2,1}$ is the directional total variation defined with the direction gradient of the previous section.

- $\left\langle c_{f}, u\right\rangle_{F}$ is the Chan et al. data fidelity term [6] where $\left(c_{f}\right)_{i, j}=\left(c_{1}-f_{i, j}\right)^{2}-\left(c_{2}-f_{i, j}\right)^{2}$ and $\langle u, v\rangle_{F}=$ $\sum_{i, j} u_{i, j} v_{i, j}$ is the Frobenius product. The scalar $c_{1}$ and $c_{2}$ are respectively the foreground and background constant and $f$ is the initial image.

\section{IMPLEMENTATION}

In this section, we provide the implementation details to compute the directional total variation and obtain the segmentation results of Sec. 4. In the following, we consider the case of a span $V=$ $\left(\mathrm{v}_{1}, \mathrm{v}_{2}, \mathrm{v}_{3}, \mathrm{v}_{4}\right)$ (i.e. $n=1$, see Fig. $\left.2 \mathrm{a}\right)$.

\subsection{Embedding the tubular structure priors}

We have seen that the directional TV requires two tubular structure priors: a vesselness feature $\Phi$ and a direction feature. Several filters 


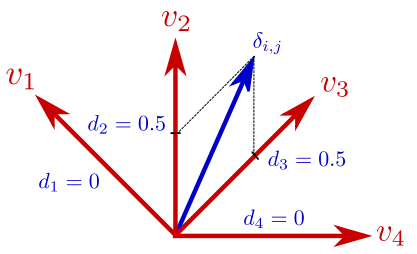

Fig. 3: Decomposition of the direction vector at pixel $(i, j), \delta_{i, j}$, on each vector of the span.

can provide these features, for instance some wavelet-based filters, the Frangi filter and the RORPO filter. In [9], we demonstrated that, thanks to a non-local path-based approach, the RORPO vesselness is a more robust tubular structures detector than Frangi's. We have also shown that the RORPO direction feature is more precise than Frangi's when applied to a grey scale image (in particular, before a segmentation step). Due to these properties, we focus on the RORPO vesselness and direction features.

In our approach, the vesselness feature $\Phi$ is kept unchanged whereas the directions coefficients $d^{q}(q \in \llbracket 1, p \rrbracket)$ must be computed from the direction feature as follows:

Let $\Delta \in X \times X$ be the normalized direction feature. $\Delta_{i, j}=$ $\left(\Delta_{i, j}^{x}, \Delta_{i, j}^{y}\right)$ is the vector that gives the local direction of the tubular structure at pixel $(i, j)$. The $\left(d^{q}\right)_{i, j}$ are the decomposition coefficients of $\Delta_{i, j}$ on the span $V$. We note that if all the $d^{q}$ have the same value, $\nabla_{D}$ is similar to a regular isotropic gradient $\nabla$. In order to obtain a real directional gradient, we impose that the decomposition results in null coefficients except for those of the two span vectors bracketing $q \in \llbracket 1, p \rrbracket$ (see Fig. 3). More formally, let $\mathrm{v}_{m}$ and $\mathrm{v}_{n}$ be the two bracketing span vectors of $\Delta_{i, j}$; then:

$$
\Delta_{i, j}=\sum_{q=1}^{p} d_{i, j}^{q} \mathbf{v}_{q}=d_{i, j}^{m} \mathbf{v}_{m}+d_{i, j}^{n} \mathbf{v}_{n}
$$

From Eq. (7), we derive the linear system:

$$
\begin{aligned}
& d_{i, j}^{m} \mathbf{v}_{m} \cdot \mathbf{v}_{m}+d_{i, j}^{n} \mathbf{v}_{n} \cdot \mathbf{v}_{m}=\Delta_{i, j} \cdot \mathbf{v}_{m} \\
& d_{i, j}^{m} \mathbf{v}_{m} \cdot \mathbf{v}_{n}+d_{i, j}^{n} \mathbf{v}_{n} \cdot \mathbf{v}_{n}=\Delta_{i, j} \cdot \mathbf{v}_{n}
\end{aligned}
$$

As we consider the span $V=\left(\mathrm{v}_{1}, \mathrm{v}_{2}, \mathrm{v}_{3}, \mathrm{v}_{4}\right), \mathrm{v}_{m} . \mathrm{v}_{n}=\frac{\sqrt{2}}{2}$ because the angle between adjacent couple $\mathrm{v}_{m}$ and $\mathrm{v}_{n}$ is $\frac{\pi}{4}$. Then we simply deduce:

$$
\begin{aligned}
& d_{i, j}^{m}=2 \Delta_{i, j} \cdot \mathrm{v}_{m}-\sqrt{2} \Delta_{i, j} \cdot \mathbf{v}_{n} \\
& d_{i, j}^{n}=2 \Delta_{i, j} \cdot \mathrm{v}_{n}-\sqrt{2} \Delta_{i, j} \cdot \mathbf{v}_{m}
\end{aligned}
$$

\subsection{Algorithm}

In [6], the Chan-Vese segmentation problem, with classical TV, was proved to be equivalent to the following convex constrained problem:

$$
\underset{u \in[0,1]^{N \times N}}{\operatorname{minimize}}\left\langle c_{f}, u\right\rangle_{F}+\lambda\|\nabla u\|_{2,1}
$$

Let us recall the proximity operator (see [17] for a tutorial on proximity operators) of a function $\varphi$ :

$$
\operatorname{prox}_{\varphi} x=\arg \min _{y \in X} \varphi(y)+\frac{1}{2}\|x-y\|_{2,2}^{2}
$$

It can be shown from [18], that the problem (8) admits a solution given by the proximal point splitting algorithm:

$$
u_{n+1}=\operatorname{prox}_{\gamma h}\left(u_{n}-\gamma c_{f}\right)
$$

where $h=\lambda\|\nabla u\|_{2,1}+\iota_{[0,1]} \times \times N(u)$ with $\gamma$ a step-size parameter and $\iota_{[0,1]} \times{ }^{N}$ the indicator function defined as follows:

$$
\iota_{[0,1]} \times \times N(u)= \begin{cases}0 & \text { if } u \in[0,1]^{N \times N} \\ +\infty & \text { otherwise }\end{cases}
$$

In [19], Beck et al. considered a dual approach to find the proximity operator of $\gamma h$. This results in a projected gradient-based algorithm.

We simply adapt this approach to our directional $\mathrm{TV}_{d}(u)$. We only show the inner loop of the algorithm:

$$
\begin{aligned}
& u_{k+1}=P_{C}\left(\mathrm{TV}_{d}\left(u_{k}\right)-\lambda \operatorname{div}\left(\xi_{k}\right)\right) \\
& \xi_{k+1}=P_{B \ell_{2}}\left(\xi_{k}+\tau \nabla_{D} u_{k+1}\right)
\end{aligned}
$$

where $P_{B \ell_{2}}$ is the projection on the $\ell_{2}$ unit balls, $P_{C}$ is the projection onto the convex set $C(C=[0,1]$ to satisfy the constraint $u \in$ $[0,1]^{N \times N}$ ) and $\nabla_{D}=D \nabla_{d}$ where $\nabla_{d}$ is defined as follows:

$$
\begin{array}{lr}
\left(\nabla_{d} u\right)_{i, j}^{1}=u_{i-1, j-1}-u_{i, j} & \text { if } i, j>0 \\
\left(\nabla_{d} u\right)_{i, j}^{2}=u_{i-1, j}-u_{i, j} & \text { if } i>0 \\
\left(\nabla_{d} u\right)_{i, j}^{3}=u_{i-1, j+1}-u_{i, j} & \text { if } i>0, j<N-1 \\
\left(\nabla_{d} u\right)_{i, j}^{4}=u_{i, j+1}-u_{i, j} & \text { if } j<N-1 \\
\left(\nabla_{d} u\right)_{i, j}^{n}=0 \quad \forall n \in \llbracket 1,4 \rrbracket & \text { otherwise }
\end{array}
$$

$\operatorname{div}$ is the divergence given by the adjoint relation $\langle-\operatorname{div} p, u\rangle_{X}=$ $\langle p, \nabla u\rangle_{Y}, \forall p \in Y=X^{4}, p=\left(p^{1}, p^{2}, p^{3}, p^{4}\right), \forall u \in X$. If we assume that $p_{N-1, N-1}^{1}=p_{N-1, j}^{2}=p_{N-1,0}^{3}=p_{i, 0}^{4}=0 \quad \forall i, j \in$ $\llbracket 0, N \llbracket$, then div is given by:

$$
\begin{array}{r}
(\operatorname{div} p)_{i, j}=p_{i, j}^{1}-p_{i+1, j+1}^{1}+p_{i, j}^{2}-p_{i+1, j}^{2} \\
+p_{i, j}^{3}-p_{i+1, j-1}^{3}+p_{i, j}^{4}-p_{i, j-1}^{4}
\end{array}
$$

\section{EXPERIMENTS AND RESULTS}

In this section, we present segmentation results on both synthetic and real data. In order to demonstrate the usefulness of our regularization term for tubular structure segmentation, we compare our results with two models: the classical Chan Eq. (8), and the weighted TV Eq. (11) segmentation models.

$$
\underset{u \in X}{\operatorname{minimize}}\left\langle c_{f}, u\right\rangle_{F}+\sum_{0 \leq i, j<N} \Lambda \sqrt{\left((\nabla u)_{i, j}^{x}\right)^{2}+\left((\nabla u)_{i, j}^{y}\right)^{2}}
$$

where $\Lambda=\frac{1}{\lambda \alpha+(\alpha-1) \Phi_{i, j}}$ is the adaptive regularization parameter with $\lambda \in \mathbb{R}$ the classical regularization parameter and $\alpha \in[0,1]$.

\subsection{Synthetic image segmentation}

We first segmented a noisy synthetic image with our directional model and the two above methods, with increasing regularization parameter $\lambda$. Results are shown in Fig. 4.

With a small value of $\lambda$, the three models results are equivalent as with a small regularization, the optimization process is dominated by the data fidelity term which is the same in the three models.

Then, when $\lambda$ increases, the line-like structures progressively disappear in the classical Chan model. The lower regularization inside line-like structure let the weighted TV better preserved them. Whereas our direction TV model with a higher and directional regularization both preserved and reconnect line-like structures resulting in the best segmentation. 


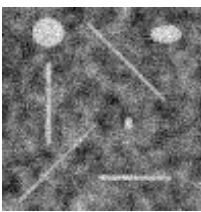

(a) Initial image (e) RORPO vesselness $(\Phi)$

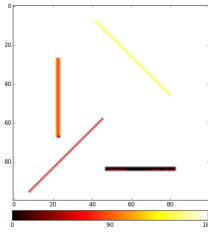

(i) RORPO directions

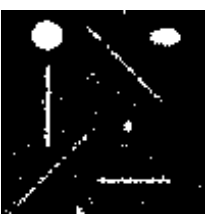

(b) $\lambda=0.1$

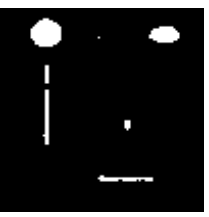

(f) $\lambda=0.6$

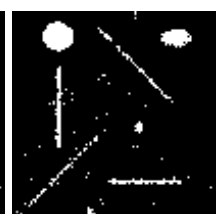

(c) $\lambda=0.1$

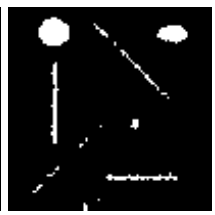

(g) $\lambda=0.6$

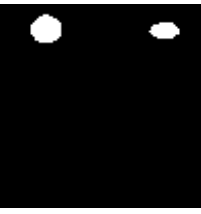

(j) $\lambda=1$

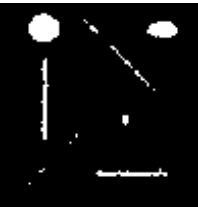

(k) $\lambda=1$

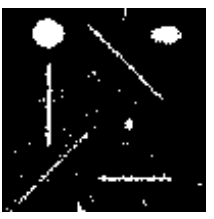

(d) $\lambda=0.1$

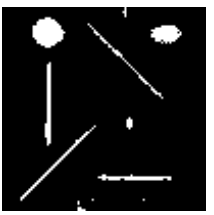

(h) $\lambda=0.6$
Fig. 4: Comparison of the classical Chan model (b, f, j) and the weighted TV model (c, g, k) with our directional TV model (d, h, 1) for increasing regularization parameter $\lambda$. The direction feature is represented only inside tubular structures, in degrees, with a colormap from $0^{\circ}$ (black) to $180^{\circ}$ (white).

\subsection{Segmentation of retinal images from the DRIVE database}

In order to quantitatively analyse our directional TV results, we segmented the 20 images of the testing set of the Drive database [20]. We optimized each parameters to have a fair study compared with the state of the art, and computed the accuracy (Acc) from true positives/negatives (TP, TN), false positives/negatives (FP, FN) inside the entire field of view (FOV). We subtracted the median filter result to the input, before each segmentation, in order to homogenize the image background, since the Chan et al. data fidelity assumes homogeneous background and foreground intensities.

The quantitative results are shown in Fig. 5. We added, for comparison, a few best state of the art results (see [23] for a recent survey). The accuracy of our method is close to state of the art methods, even though we do not propose a dedicated segmentation model for retinal images, but only a generic directional regularization term for tubular structures. This regularization may be embedded in more complex variational framework such as [24], instead of the classical $\mathrm{TV}$, to improve results. The accuracy and TP of our method are still higher than that of the classical Chan et al. . This confirms that our

\begin{tabular}{|l|c|c|c|}
\hline & TP & TN & Acc \\
\hline Chan & 0.656 & 0.985 & 0.9421 \\
Proposed segmentation & 0.690 & 0.981 & 0.9434 \\
Staal [20] & - & - & 0.9442 \\
Lupascu [21] (supervised method) & 0.6728 & 98.74 & 0.9597 \\
Al-Rawi [22] (matched filtering) & - & - & 0.9535 \\
Human observer & - & - & 0.9470 \\
\hline
\end{tabular}

Fig. 5: Quantitative segmentation results on the DRIVE database.

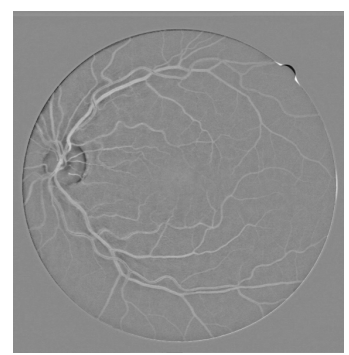

(a) Background corrected initial image

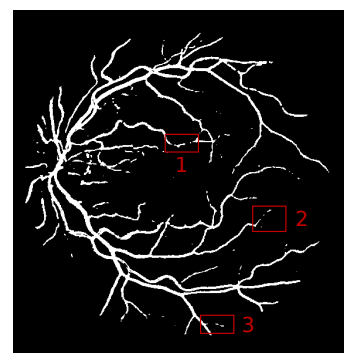

(c) Chan model

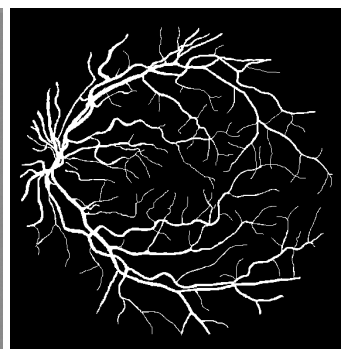

(b) Ground truth

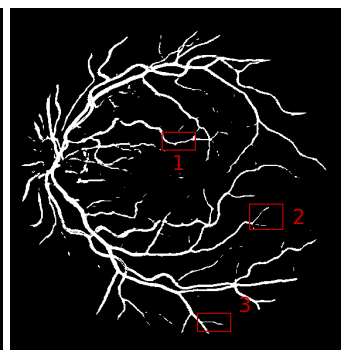

(d) Proposed model

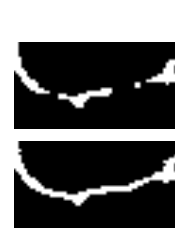

(e) box 1

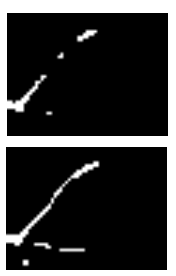

(f) box 2

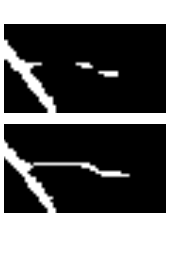

(g) box 3

Fig. 6: Comparison of the classical Chan et al. model (c) with our directional TV model (d) on the first DRIVE image (a) and its ground truth (b). (e-g) zoom on a few extremities of blood vessels of the Chan model results (top) and our proposed model results (bottom).

directional TV effectively improves the tubular structure segmentation. Fig. 6 shows a visual comparison of the Chan segmentation and ours on the first DRIVE image. A zoom on a few blood vessels extremities show that we successfully reconnect most small vessels. As these reconnections represent only a few pixels within the image, the accuracy of both methods does not appear quantitatively different even if the improvement is real and significant. Indeed a connected network is a much desired feature in blood vessels segmentation.

\section{CONCLUSION AND FUTURE WORK}

In this article, we have proposed a new regularization term for variational problems suitable for tubular structures: the directional total variation. We have shown on both synthetic and real data that this directional TV enhances the segmentation results by regularizing inside tubular structures solely along their local axis. Thanks to robust directional information, it tends to reconnect noisy tubular structures segments and to better preserve their extremities. In addition to segmentation, this directional regularization term should be useful in other variational problems such as denoising, debluring, inpainting, and others. Directional TV with larger spans are also interesting to consider for noisier applications as these should improve the reconnections of tubular structures segments. 


\section{REFERENCES}

[1] O. Tankyevych, H. Talbot, N. Passat, M. Musacchio, and M. Lagneau, "Angiographic image analysis," in Medical Image Processing: Techniques and Applications, Geoff Dougherty, Ed., pp. 115-144. Springer, 2011.

[2] H. Talbot, Oriented patterns in image analysis: from thin objects to flow-based methods, Habilitation Thesis, Université Paris-Est, 2013, https: / / hal .archives-ouvertes. fr/tel-01099256v1.

[3] A.N. Tikhonov, "Regularization of incorrectly posed problems," Soviet Math Dokl, vol. 4, no. 6, pp. 1624-1627, 1963.

[4] L.I. Rudin, S. Osher, and E. Fatemi, "Nonlinear total variation based noise removal algorithms," Phys D, vol. 60, no. 1-4, pp. 259-268, 1992.

[5] T.F. Chan and Vese L.A., "Active active contours without edges," IEEE Trans. Image Process., vol. 10, no. 2, pp. 266277, 2001.

[6] T.F. Chan, S. Esedoglu, and M. Nikolova, "Algorithms for finding global minimizers of image segmentation and denoising models," SIAM J. Appl. Math., vol. 66, pp. 1632-1648, 2006.

[7] A.F. Frangi, W.J. Niessen, K.L. Vincken, and M.A. Viergever, "Multiscale vessel enhancement filtering," in Proc. Med. Image Computing \& Comp.-Assist. Intervention (MICCAI). 1998, vol. 1496 of Lect. Notes Comput. Sci., pp. 130-137, Springer.

[8] O. Merveille, H. Talbot, L. Najman, and N. Passat, "Tubular structure filtering by ranking orientation responses of path operators," in Proc. Eur. Conf. Comput. Vis. 2014, vol. 8690 of Lect. Notes Comput. Sci., pp. 203-218, Springer.

[9] O. Merveille, H. Talbot, L. Najman, and N. Passat, "Tubular structure analysis by ranking the orientation responses of path operators," 2016, https://hal. archives-ouvertes.fr/hal-01262728.

[10] J.A. Tyrrell, E. di Tomaso, D. Fuja, Ricky Tong, K. Kozak, R.K. Jain, and B. Roysam, "Robust 3-D modeling of vasculature imagery using superellipsoids," IEEE Trans. Med. Imag., vol. 26, no. 2, pp. 223-237, 2007.

[11] C. Tai, X. Zhang, and Z. Shen, "Wavelet frame based multiphase image segmentation," SIAM J. Imaging Sci., vol. 6, no. 4, pp. 2521-2546, 2013.

[12] R. Rigamonti and V. Lepetit, "Accurate and efficient linear structure segmentation by leveraging ad hoc features with learned filters," in Proc. Med. Image Computing \& Comp.Assist. Intervention (MICCAI). 2012, vol. 7510 of Lect. Notes Comput. Sci., pp. 189-197, Springer.

[13] N. Y. El-Zehiry and L. Grady, "Vessel segmentation using 3D elastical regularization," in Proc. IEEE Int. Symp. Biomed. Imaging, 2012, pp. 1288-1291.

[14] O. Miraucourt, A. Jezierska, H. Talbot, S. Salmon, and N. Passat, "Variational method combined with Frangi vesselness for tubular object segmentation," Comput. Math. Biomed Eng., pp. 485-488, 2015.

[15] G. Peyré, S. Bougleux, and L. Cohen, "Non-local regularization of inverse problems," in Proc. Eur. Conf. Comput. Vis. 2008, vol. 5304 of Lect. Notes Comput. Sci., pp. 57-68, Springer.
[16] C. Couprie, L. Grady, L. Najman, J.C. Pesquet, and H. Talbot, "Dual constrained tv-based regularization on graphs," SIAM J. Imaging Sci., vol. 6, no. 3, pp. 1246-1273, 2013.

[17] P.L. Combettes and J.C. Pesquet, "Proximal splitting methods in signal processing," in Fixed-Point Algorithms for Inverse Problems in Science and Engineering, H. Heinz Bauschke, S. Regina Burachik, L. Patrick Combettes, Veit Elser, Russell D. Luke, and Henry Wolkowicz, Eds., pp. 185-212. Springer Verlag, New York, 2011.

[18] P.L. Combettes and V.R. Wajs, "Signal recovery by proximal forward-backward splitting," Multiscale Model. Simul., vol. 4, pp. 1168-1200, 2005.

[19] A. Beck and M. Teboulle, "Fast gradient-based algorithms for constrained total variation image denoising and deblurring problems," IEEE Trans. Image Process., vol. 18, no. 11, pp. 2419-2434, 2009.

[20] J.J. Staal, M.D. Abramoff, M. Niemeijer, M.A. Viergever, and B. van Ginneken, "Ridge based vessel segmentation in color images of the retina," IEEE Trans. Med. Imag., vol. 23, no. 4, pp. 501-509, 2004.

[21] C. A. Lupaşcu, D. Tegolo, and E. Trucco, "Fabc: retinal vessel segmentation using adaboost," IEEE Trans. Inform. Tech. Biomed., vol. 14, no. 5, pp. 1267-1274, 2010.

[22] M. Al-Rawi, M. Qutaishat, and M. Arrar, "An improved matched filter for blood vessel detection of digital retinal images," Comput. Biol. Med., vol. 37, no. 2, pp. 262-267, 2007.

[23] M. M. Fraz, P. Remagnino, A. Hoppe, B. Uyyanonvara, A. R. Rudnicka, C. G. Owen, and S. A. Barman, "Blood vessel segmentation methodologies in retinal images-a survey," Comput. Meth. Programs Biomed., vol. 108, no. 1, pp. 407-433, 2012.

[24] A. Jezierska, O. Miraucourt, H. Talbot, S. Salmon, and N Passat, "A non-local Chan-Vese model for sparse, tubular object segmentation," in Proc. Int. Conf. Image Process., 2014, pp. 907-911. 